\title{
Resonant slow extraction with constant optics for improved separatrix control at the extraction septum
}

\author{
V. Kain $\odot,{ }^{*}$ F. M. Velotti, M. A. Fraser, B. Goddard, J. Prieto, and L. S. Stoel \\ CERN, Geneva, Switzerland \\ M. Pari \\ University of Padova and INFN, Padova, Italy
}

(Received 27 May 2019; published 9 October 2019)

\begin{abstract}
Losses and component activation are limiting performance factors for slow extraction with high-power applications, and new techniques of loss-reduction, such as bent crystals, require a stable and narrow separatrix angular spread. Conventional tune-sweep slow extraction results in an optics change and an accompanying separatrix rotation through the spill. This can be compensated by a dynamic closed-orbit bump, but requires a high level of complexity for setting up and monitoring. For the Super Proton Synchrotron (SPS), a simpler and powerful new extraction technique has been developed and deployed, providing a mechanism to fix the machine optics and hence separatrix completely through the spill. The technique with the name constant optics slow extraction (COSE) relies on high chromaticity and scaling all machine settings with beam rigidity following the momentum distribution of the beam. In this paper we describe the new COSE concept and its successful operational deployment in the SPS during the 2018 run.
\end{abstract}

DOI: $10.1103 /$ PhysRevAccelBeams.22.101001

\section{INTRODUCTION}

The method of slow extraction allows to extract beam from circular accelerators in long spills of seconds by exploiting the separatrices of the third order resonance. Long spills are of interest for fixed target high energy physics experiments and also for medical irradiation facilities. The extraction equipment typically consists of an electrostatic septum (ES) with thin wires or foils as anode material followed by other thicker septa in the extraction channel until the beam is separated sufficiently from the circulating beam at the entrance of a transfer line. With slow extraction, a fraction of the extracted particles unavoidably hits the septum wires or foil. The created residual radioactivation of the ES and downstream equipment has an impact on equipment lifetime and hands-on maintenance strategies. In fact the upper limit of intensity extracted per year through the same extraction channel is defined by the maximum allowable activation of equipment given a certain maintenance strategy. For the $400 \mathrm{GeV} / \mathrm{c}$ SPS, beam loss at the ES is one of the main performance limitations for the present SPS fixed target physics program

\footnotetext{
*verena.kain@cern.ch

Published by the American Physical Society under the terms of the Creative Commons Attribution 4.0 International license. Further distribution of this work must maintain attribution to the author(s) and the published article's title, journal citation, and DOI.
}

and for any future experiments requiring large numbers of protons on target (POT).

Presently slow extraction beam losses are in the order of a few \% at the SPS ES [1]. Methods of reducing these losses and mitigating their effects are therefore an active study topic for CERN's Physics Beyond Colliders program [2]. Various promising techniques to reduce slowextraction losses by means of, for example, bent silicon crystals [3], diffusers [4] or phase space folding with multipoles [5] have been proposed. All these benefit in efficiency from a constant phase space representation, i.e., constant separatrix angle, throughout the spill or constant optics in the case of exploiting multipoles for loss reduction. The angular acceptance of crystals aligned to channel is limited to values similar to the intrinsic separatrix width. During the 2018 SPS run the authors developed an elegant and operationally inexpensive method with the name constant optics slow extraction to fix the optics and the separatix angle at the septum throughout the SPS slowextracted spill, without the complication of controlling and setting up dynamic bumps or other means of correction and without the need for additional hardware or electronics in the accelerator.

\section{SLOW EXTRACTION AT THE CERN SUPER PROTON SYNCHROTRON}

The third order resonant extraction from long straight section II (LSS2) at the $\approx 7 \mathrm{~km}$ circumference SPS at 
CERN serves the fixed target experiments in the SPS North Area [6].

A set of sextupoles are set to $k_{2}=-0.12 \mathrm{~m}^{-3}$ at the top energy of $400 \mathrm{GeV}$ to create a stable area in the horizontal phase space of the beam. The machine tune is then moved towards $Q_{h}=262 / 3$ to drive the slow extraction. The typical spill duration is $4.8 \mathrm{~s}$. To reduce the effect of power supply ripple on degrading the quality of the spill, the normalized chromaticity is set large to $\xi_{x}=-1$ during slow extraction and the beam momentum spread increased to $\frac{\delta p}{p}= \pm 1.5 \times 10^{-3}$ before the $\mathrm{rf}$ is switched off. The particles with phase space coordinates outside the stable region move away from it along the third order resonance separatrices until they enter into the field of the long $(17 \mathrm{~m})$ multiunit ES which deflects them into the extraction channel consisting of eight magnetic septa. A horizontal orbit bump is used in addition to bring the circulating beam close to the ES and control the horizontal size of the extracted beam. Note that the Hardt condition [7] cannot be fulfilled at the SPS ES such that the separatrices of particles with different momenta and amplitudes have different angles at the septum location.

For an ideal spill, the rate of extracted particles $d N / d t$ should remain constant along the extraction flat-top. In the past, the intensity signal from the beam extracted to the North Area transfer lines was compared to a reference value and used in a feed-back loop with the dedicated servoquadrupole system consisting of 4 short QMS quadrupoles installed in cell 116 [8]. The servo quadupole feed-back was in use until end of 2014, when it was replaced by a feed-forward algorithm using the main quadrupoles [9]. In 2018, this was again improved by adapting the algorithm to use all magnetic elements in the SPS lattice, with constant optics slow extraction (COSE).

With the conventional technique of quadrupole driven slow extraction in the SPS, the optics and hence beta functions, natural chromaticity and the phase advance between extraction sextupoles and septum all change as a function of the time on the extraction flat-top. The effect on the separatrix presentation at the location of the ES in normalized phase space from simulation is shown in Fig. 1, where the different colors indicate the separatrices for particles with different momenta, which in the SPS is a proxy for time in the spill, since particles with the lowest momentum are extracted first. The extent of the optics variation can be visualized by calculating the resonance driving term, or the equivalent virtual sextupole [10]. The optics variation results in a change of amplitude and phase of the virtual sextupole as a function of extracted particle momentum (or equivalently time), see Fig. 2.

Dynamic bumps [11] in position $x$ and angle $x^{\prime}$ at the ES to compensate the change in separatrix presentation were considered to reduce the angular spread. A series of parametric simulations were performed to evaluate the expected loss reduction [12]. The results are summarized

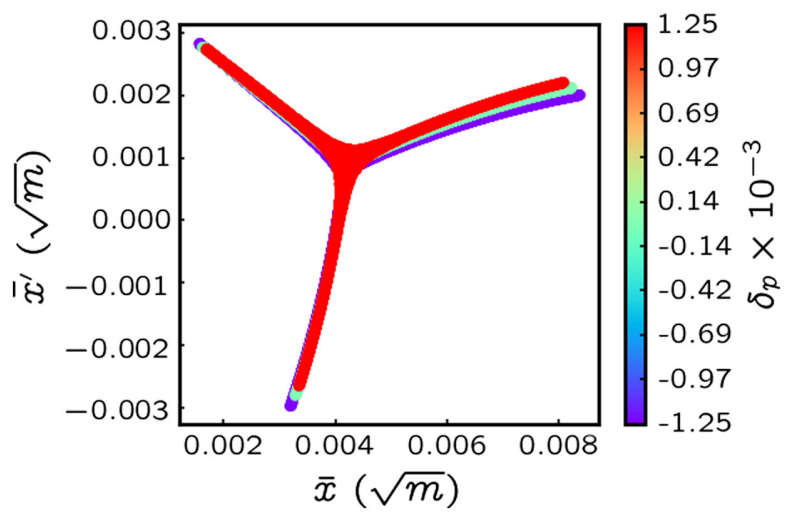

FIG. 1. Change of separatrix presentation at the ES during the spill for SPS quadrupole driven slow extraction with main quadrupoles (tune sweep). The different colors indicate the separatrices for different momenta.

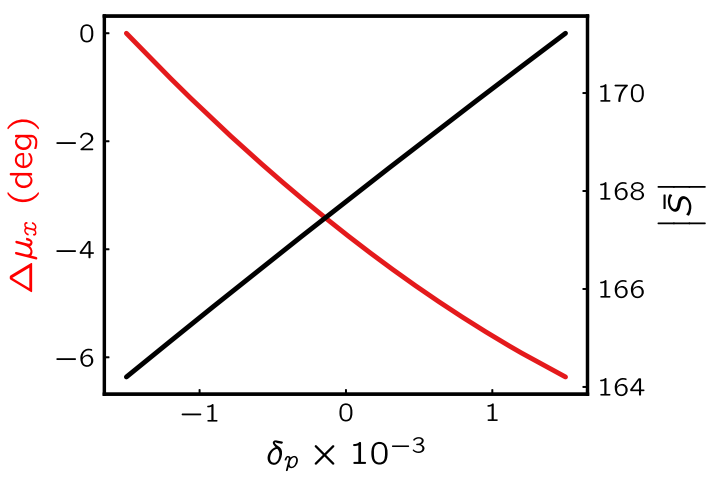

FIG. 2. Variation of the amplitude and phase of the virtual sextupole (driving term) during the spill with the SPS tune sweep slow extraction.

in Fig. 3, where the expected loss reduction as a function of ES effective thickness is shown. Note that the expected loss reduction is in the order of a few percent only for an ES equivalent thickness above $300 \mu \mathrm{m}$. From measurements in 2018 with both passive and active diffusers the effective thickness was estimated to be in the order of $500 \mu \mathrm{m}[3,4]$.

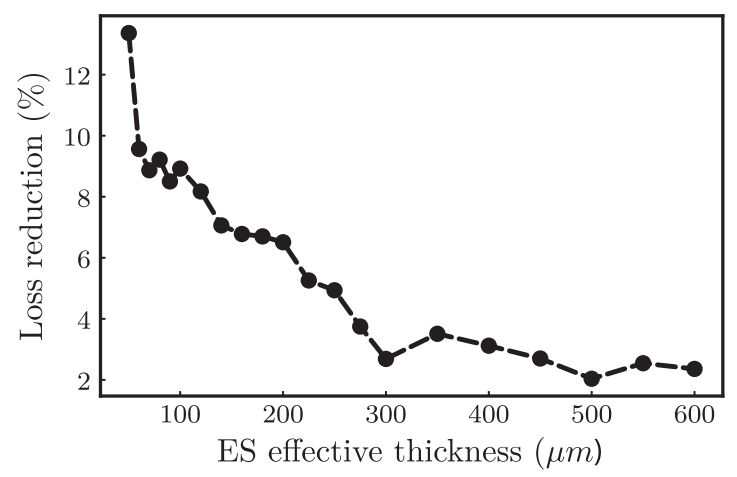

FIG. 3. Simulated loss reduction at SPS ES with dynamic bump as function of electrostatic septum effective thickness in $\mu \mathrm{m}$. 
Hence, no significant loss reduction should be expected from the reduction of the angular spread alone at the ES. Implementation details and setting up strategy for a dynamic extraction bump were complex and were never finalized as they became redundant with the new approach of constant optics slow extraction.

\section{CONSTANT OPTICS SLOW EXTRACTION}

The basic idea behind COSE is to exploit the fact that the separatrix change during slow extraction is the consequence of moving the resonance to select the particles which should be extracted via their momenta. Therefore, instead of moving the resonance, the machine settings could instead be scaled according to the momentum to be extracted, while the resonance could be kept fixed. Figure 4 illustrates the COSE idea with a Steinbach diagram. It looks similar to the betatron core slow extraction, with the only difference that for COSE the momentum of the extracted beam is not constant. However, it shares the attractive feature that the machine optics is completely constant throughout the extraction, since all machine elements are scaled according to

$$
\frac{\delta^{n} B(t)}{\delta x^{n}}=k_{n} \cdot B \rho(t)
$$

where the normalized mutlipole strength $k_{n}$ is constant, $t$ is the time during the extraction process and $B \rho$ the beam rigidity that changes with time. Note that COSE is a chromatic extraction and hence requires large chromaticity.

Figure 5 shows the comparison of the extracted beam distribution at the ES from simulation between standard quadrupole driven extraction (top) and COSE (bottom). The correlation between $x^{\prime}$ and momentum of the extracted particles disappears and the local angular spread at the electrostatic septum is reduced by $\approx 20 \%$ (Fig. 6). Figure 7 shows the simulation of the evolution of the resonance

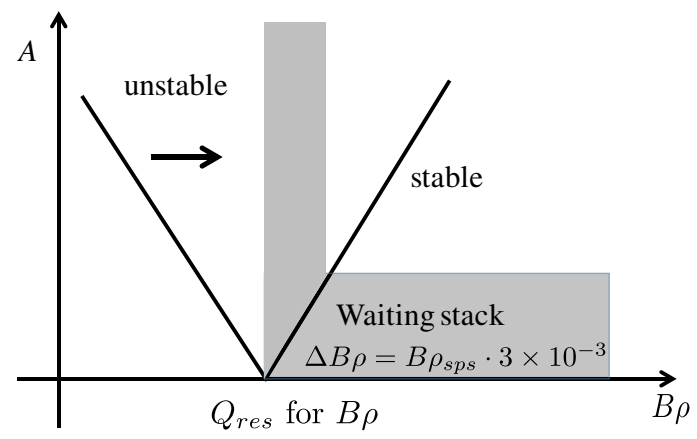

FIG. 4. Steinbach diagram to illustrate COSE slow extraction process: At arrival at the extraction flat-top the tune is set to the resonant one for the lowest momentum particles. Then the beam rigidity $B \rho$ (virtual scaling parameter of magnetic settings) is increased until all particles are extracted. The assumed total momentum spread in the illustration is $\delta p / p=3 \times 10^{-3}$.
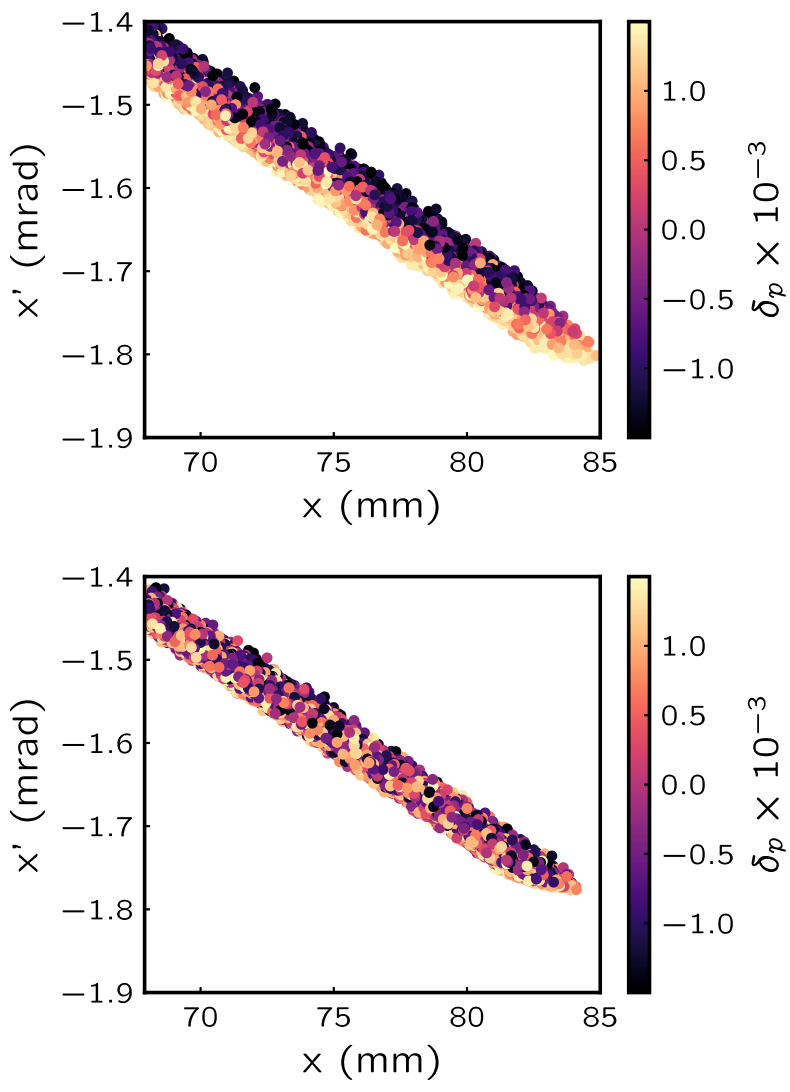

FIG. 5. Phase space plot of extracted beam at SPS ES entrance for entire spill from simulation. The upper plot was obtained with conventional tune sweep extraction and the lower plot with COSE. The correlation between $x^{\prime}$ and particle momentum disappears for COSE and the angular spread at the ES is reduced.

driving term during the extraction with COSE; the optics stays frozen throughout the spill.

The SPS accelerator equipment settings management based on the Large Hadron Collider Software Architecture (LSA) [13] offers the means for driving slow extraction through high level parameters such as $B \rho$ or momentum and is discussed in the Appendix. The other vital ingredient

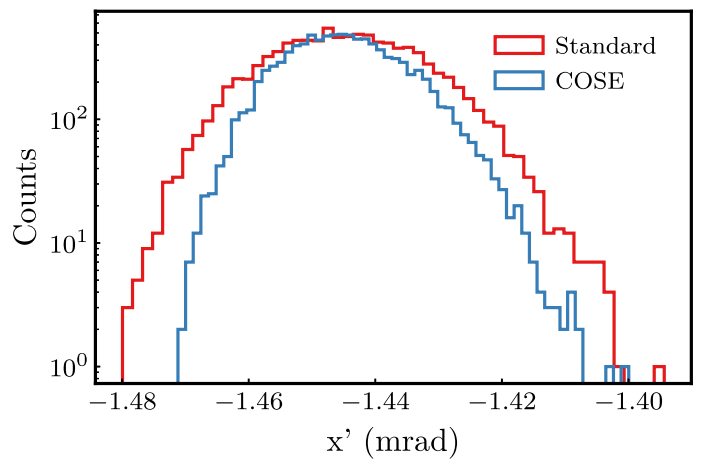

FIG. 6. Angular distribution at the location of the ES wires for COSE (red, $\sigma=9.8 \mu \mathrm{rad}$ ) and tune sweep (Standard, blue; $\sigma=12.3 \mu \mathrm{rad})$. 


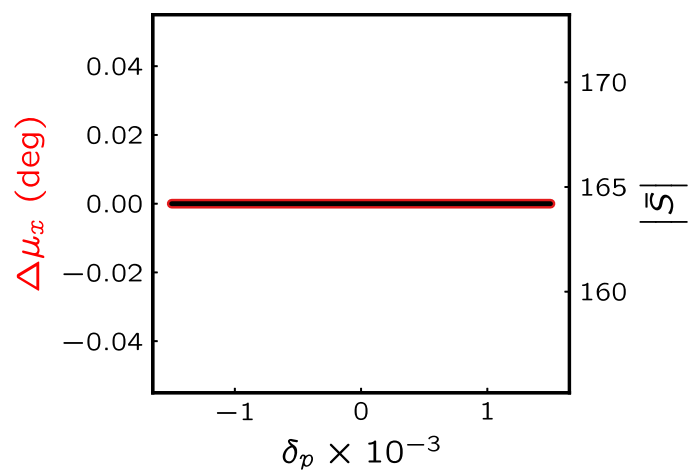

FIG. 7. Variation of amplitude and phase of virtual sextupole during spill with COSE.

for COSE to become operationally interesting was the possibility of feed-forward spill control with the Autospill algorithm [9]. Details on its implementation are also described in the Appendix.

\section{RESULTS OF DEPLOYING COSE IN OPERATION}

The first step for setting up COSE is to define the required value of tune during the extraction plateau, since the tune function remains unchanged throughout extraction. The tune is adjusted to be the resonant one for the lowest momentum particles when arriving at the extraction plateau. As these particles should be on-momentum, the beam is accelerated to the flat-top design momentum (400 GeV/c) plus $\frac{1}{2} \delta p / p$, where $\delta p / p$ is the total momentum spread. This is done by radially steering the beam before the $\mathrm{rf}$ is switched off. For the rest of the extraction plateau only the high level momentum parameter (in the SPS the momentum parameter is used instead of $B \rho$ ) is changing, i.e., it is increased according to the beam momentum spread until all particles are extracted. The Autospill algorithm is applied to ensure constant spill for a given momentum distribution. It computes the required momentum function changes $d p / d t$ for constant $d N / d t$ and reconstructs a modified momentum function accordingly. The resulting functions for the SPS high level (momentum, tune) and low level (currents) parameters as calculated by the control system are shown in Figs. 8 and 9, as compared to the tune sweep extraction (Standard). While the horizontal tune parameter is fixed all along the extraction, the quadrupole currents follow the momentum distribution of the extracted particles. The vertical offset in the quadrupole currents between COSE and quadrupole driven extraction visually emphasizes the COSE methodology, where the tune at the start of slow extraction is adjusted to be the resonant one for a particle with momentum offset $\delta_{p}=0$ and $p=400 \mathrm{GeV} / \mathrm{c}-\mathrm{a}$ setting that is reached only at roughly the middle of the extraction plateau with quadrupole driven extraction.

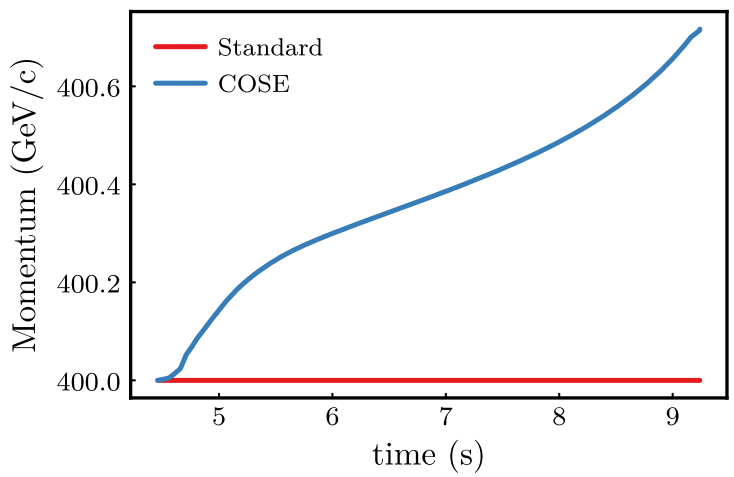

FIG. 8. Comparison of tune sweep (Standard) and COSE momentum function at the $400 \mathrm{GeV} / \mathrm{c}$ SPS flat-top. With COSE the required momentum function evolution follows the momentum distribution of the beam.

It is not straightforward to verify with measurement that the slow extracted separatrix presentation is indeed constant over time as expected with COSE, since there is no direct measurement of the separatrix presentation at the ES. From simulations a reduction of the angular spread at the ES of $\approx 20 \%$ is expected (Fig. 6). The results of the simulated distributions at the ES tracked along the transfer line to the North Area targets gave a reduction of typically $10 \%$ in horizontal beam size. Secondary emission monitor grids are available at various location in the transfer line.
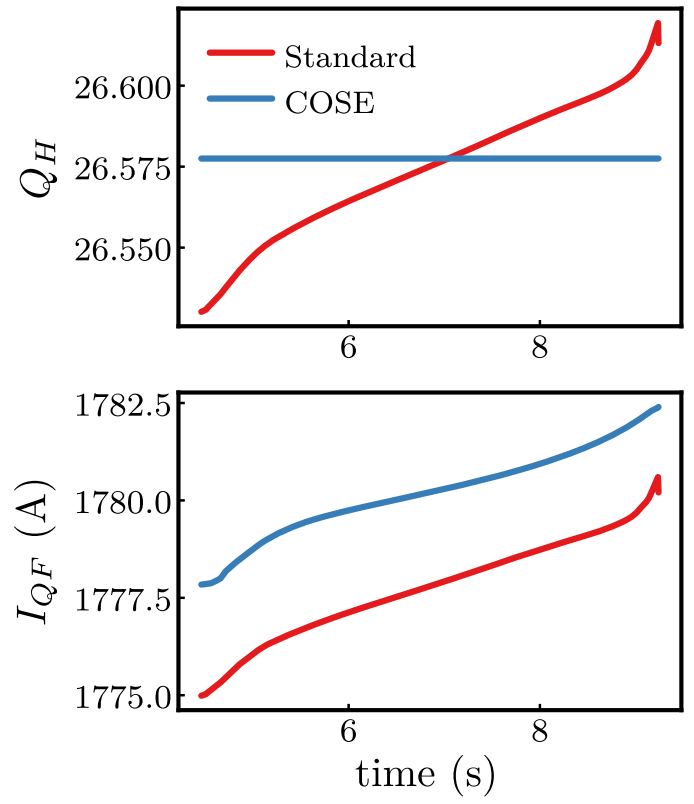

FIG. 9. Comparison of tune function on SPS flat-top for tune sweep and COSE slow extraction. The scaling with beam momentum is only visible on the current. The vertical offset in the quadrupole currents between COSE and quadrupole driven extraction corresponds to the tune difference $\Delta Q_{h}=\frac{1}{2} \delta p / p \cdot Q_{h}^{\prime}$, where $\delta p / p$ is the total initial momentum spread and $Q_{h}^{\prime}$ is the chromaticity. 


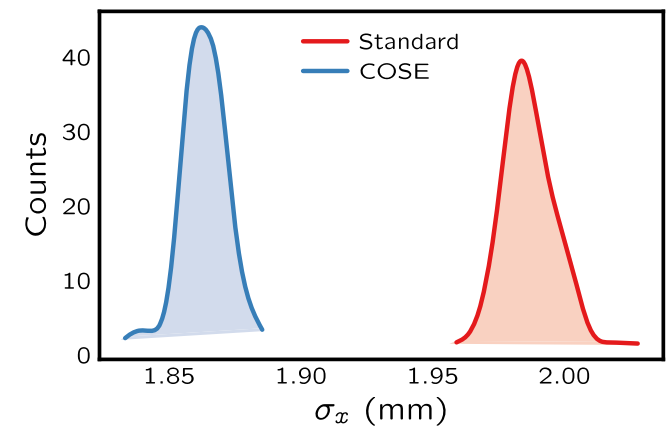

FIG. 10. Comparison of distribution of measured beam size in the transfer line to the North Area targets for tune sweep (standard) slow extraction and COSE at grid BSGH.210350.
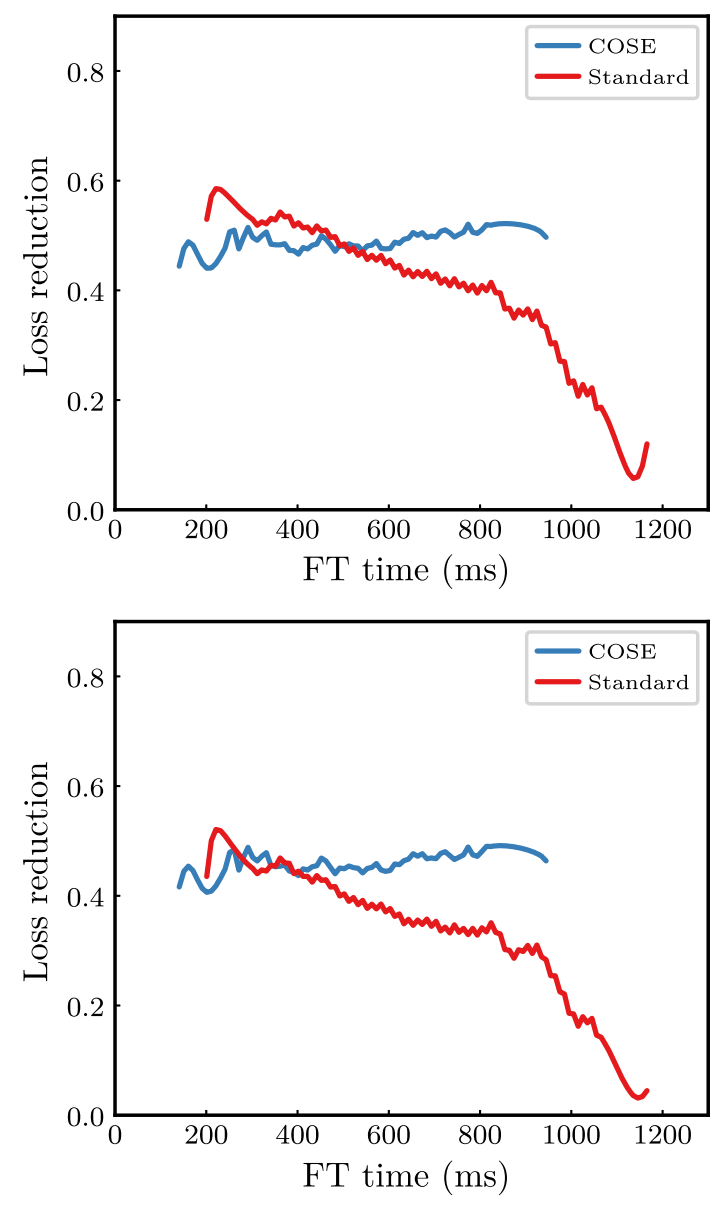

FIG. 11. Loss reduction with a bent crystal in LSS2 as function of time during slow extraction reconstructed from LHC type beam loss monitors at the peak loss location electrostatic septum ZS3 and crystal: The crystal was aligned to shadow the ES wires. As with tune sweep the separatix angle at the ES changes, the loss reduction is not constant over time and drops rapidly when reaching the edges of the crystal's angular acceptance. With COSE on the other hand the loss reduction is constant within the noise level during slow extraction. (Unfortunately the spills for COSE and tune sweep had not been adjusted to the same length during the experiment. The discussed effects are however clearly demonstrated.)
The measurements confirm the reduction in beam size at locations with favorable phase advance, see Fig. 10.

The most direct proof of the effectiveness of COSE was obtained during a test of achievable loss reduction in LSS2 with a bent silicon crystal when aligned correctly to shadow the ES wires [3]. Due to the small angular acceptance of the bent crystal in channeling, this device can be used to probe the time variation of the angular spread of the extraction separatrix. Beam losses at the crystal and the ES were recorded as a function of time and loss reduction calculated. The measurement was carried out with the conventional tune sweep slow extraction and then repeated with COSE for a $1.2 \mathrm{~s}$ long flat-top (FT). The crystal does not move during the spill. It is aligned with one position and angle for the entirety of the extraction flat-top. Figure 11 shows the results. As expected, the loss reduction changes as a function of time for slow extraction with tune sweep as the separatrix moves, while it is constant within the noise level when COSE is used. The channeling efficiency of the crystal starts to rapidly drop when the beam angular spread reaches the edges of the crystal angular acceptance.

COSE had been developed in machine development periods in the summer of 2018 and put into operation on 20th of September, 2018, for the remaining proton as well as $\mathrm{Pb}^{82+}$ fixed target run. No degradation of spill quality nor other operational issues were encountered. Figure 12 compares extraction losses between tune sweep and COSE and Fig. 13 finally shows a typical spill with COSE. No significant differences in losses over a long period have been observed, although the measurements shown in Fig. 12 may suggest a slight reduction.

The main purpose of COSE is to keep the resonance fixed over time during the slow extraction plateau and hence the separatrix. It however also offered other practical advantages. One of them is that the movement of the

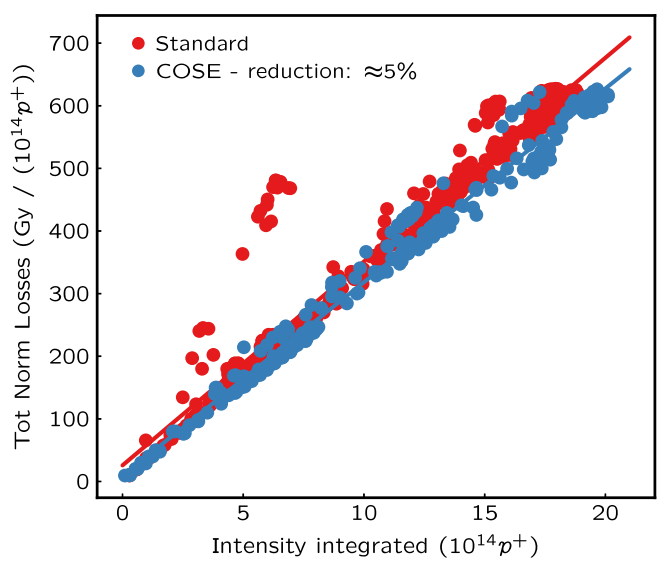

FIG. 12. Comparison of total normalized losses in LSS2 per 15 minutes as a function of intensity for tune sweep and COSE. The origin of the phenomenon of higher normalized losses for higher total intensity extracted per time unit is still under investigation. It is however present for both methods of slow extraction with a slightly reduced slope for COSE. 


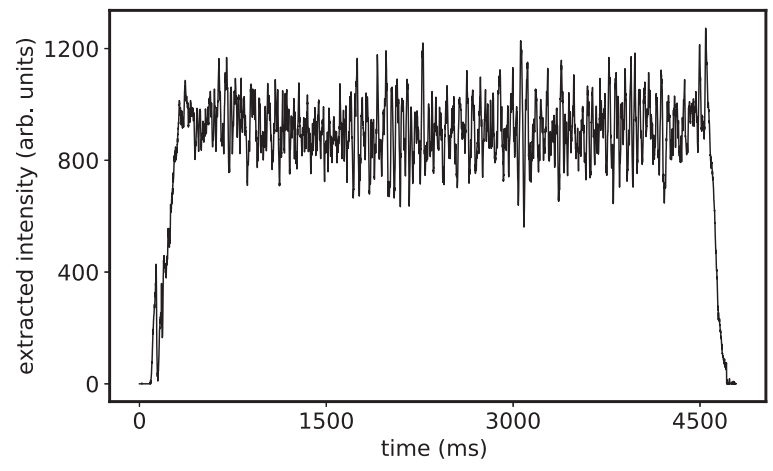

FIG. 13. Randomly chosen slow extracted proton spill to the North Area with COSE (4th of November, 2018).

trajectory in the transfer line to the North Area experimental hall and to the targets with beam energy due to dispersion can now be perfectly compensated by a simple trim of the transfer line $B \rho$ calculated by Autospill from the ring measurements. This will lead to effectively reduce the spot size where the dispersion is nonzero to only the one corresponding to the momentum spread given by the resonance stop-band width.

\section{CONCLUSION}

Sweeping the tune is perhaps the only practical means to drive slow extraction for synchrotrons with high $B \rho$, but for these high-power machines, losses at the electrostatic septum limit performance. With tune sweep, the optics is changed during the slow extraction process and with it the presentation of the beam separatrix at the electrostatic septum, which is detrimental for various loss mitigation schemes. The method constant optics slow extraction (COSE) has been developed at the $400 \mathrm{GeV} / \mathrm{c}$ CERN SPS to overcome these limitations. COSE relies on an accelerator control system based on higher level physics parameters and sufficient reproducibility of the machine to control the slow extracted spill in feed-forward. It exploits the existing hardware. The machine settings at the starting point are those to extract the lowest momentum particles, then the beam rigidity $B \rho$ is increased following the momentum distribution of the beam and all machine settings are recalculated accordingly while keeping the momentum of the beam unchanged. Dynamic bumps are not necessary to compensate for changes in the separatix position and angle. The advantage of the COSE method is that the optics, resonance, separatrix etc. all remain fixed throughout the process of slow extraction and it enables more sophisticated methods of loss reduction which rely on small overall angular separatrix spread.

Constant optics slow extraction was tested during the summer 2018 and successfully used in operation for the remaining months of the 2018 SPS fixed target physics run with protons and $\mathrm{Pb}^{82+}$. It is now the SPS operational standard for slow extraction.

\section{APPENDIX A: SETTINGS MANAGEMENT AT THE SPS}

For the largest machines at CERN, the SPS and the Large Hadron Collider, with thousands of magnets and other components, higher level, deterministic parameter control is mandatory. The LSA framework is based on a database that centralizes all hardware information, i.e., calibration curves to calculate the required current $I$ for a given field $B$. The settings of the various magnets are prepared in normalized strength defined for one or several optics associated with a so-called cycle. One of the key ingredients of the cycle is the generation of the beam rigidity $B \rho$ or momentum function (array of tuples of $B \rho$ or momentum values and time), which defines at which energy/momentum beam is injected, to which energy/ momentum the beam is accelerated, with which energy ramp rate and hence over which time. All other functions for magnets and $\mathrm{rf}$ are derived from the $B \rho$ or momentum function as well as optics and beam information. The settings are stored in the LSA database and JAVA interfaces are available to work with them. All algorithms work with normalized strengths and LSA takes care of calculating the hardware settings accordingly. The tune function for example is a so-called knob on top of the $k$-functions of the main focussing and defocussing quadrupole families in the SPS. The required change of $k_{Q F}$ and $k_{Q D}$ for a particular $\Delta Q_{h}$ is stored in the database for a given optics. Only $Q_{h}$ is modified for a required tune change in the horizontal plane. The new $k_{Q F}$ and $k_{Q D}$ functions are calculated automatically by LSA, the same is true for the required currents in the quadrupoles. Figure 14 illustrates the parameter tree for the simple case of a $Q_{h}$ knob. The arrows in the figure indicate

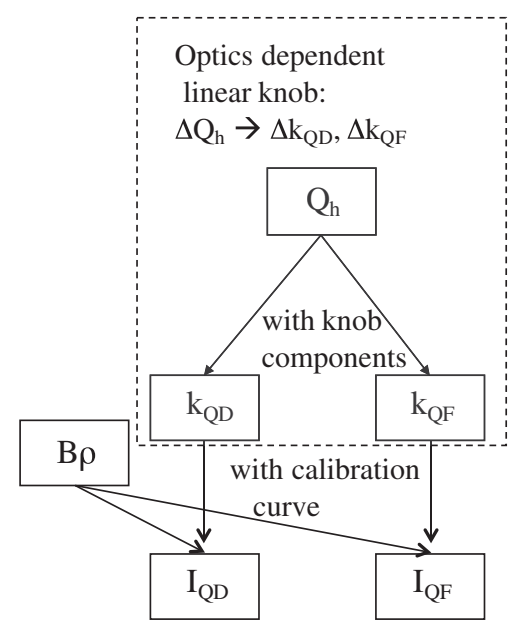

FIG. 14. Illustration of parameter tree for simple example of linear $Q_{h}$ knob. The parameter relations are stored in the database as well as the settings. So-called "makerules" are invoked when parent parameters are modified and all dependent parameters are automatically calculated following these rules. Which "makerules" to use is stored with the parameter relations in the database. 
so-called "makerules"-JAVA code that combines settings and calculates dependent settings from parent parameters. The parameter relations are stored in the database.

\section{APPENDIX B: FEED-FORWARD CONTROL OF SLOW EXTRACTED SPILL AT THE SPS}

The servo-quadrupole feedback system had to be decommissioned when the SPS began to be operated with many different magnetic cycles for different applications and with dynamic economy for energy saving (where the magnets ramp to reduced field if no beam is injected). This was because hysteresis effects required different servo-quadrupole excitation from cycle to cycle, which impacted the horizontal steering in the transfer lines to the North Area experimental hall through feed-down. For the 2015 SPS run, a new concept of spill correction was then implemented in the SPS control system in the form of Autospill, a feed-forward algorithm in a control room application, which is executed on demand. The SPS was still run with quadrupole driven slow extraction, but the tune was corrected directly through the function sent to the main quadrupoles all around the SPS with a much reduced effect on the transfer line trajectory.

In Autospill the calculation of the required tune adjustment is based on the intensity measured by the ring beam current transformers instead of the extracted intensity measured in the extraction line as for the servo-quad feedback system. For the extracted intensity $d N / d t$ to be constant, the ring intensity needs to decay linearly. No absolute reference for $d N / d t$ is defined in the algorithm. The optimum extracted intensity rate is defined by the ring intensity at the start of the slow extraction and the fact that the spill should last for the whole cycle flat-top of roughly $5 \mathrm{~s}$.

The extracted intensity rate is sampled along the extraction flat-top and translated into a required extracted intensity rate change which in turn corresponds to the required $\dot{Q}_{h}$ change according to the following relation:

$$
\frac{d N}{d t} \propto \frac{d Q_{h}}{d t}
$$

The programmed high level horizontal tune function is then recalculated according to the required $\dot{Q}_{h}$ changes.

The LSA control system of the SPS then takes care of defining the required $\mathrm{QF}$ and $\mathrm{QD}$ currents from this function according to the ring optics. Figure 15 shows an example of the horizontal tune function after Autospill correction which varies according to the momentum distribution of the beam. The dashed line indicates the programmed tune function before Autospill was available and where the constant extracted intensity was guaranteed by the servo quadrupoles.

The programmed tune functions were only re-calculated if the sequence of magnetic cycles played by the SPS changed significantly i.e., if LHC cycles were added that

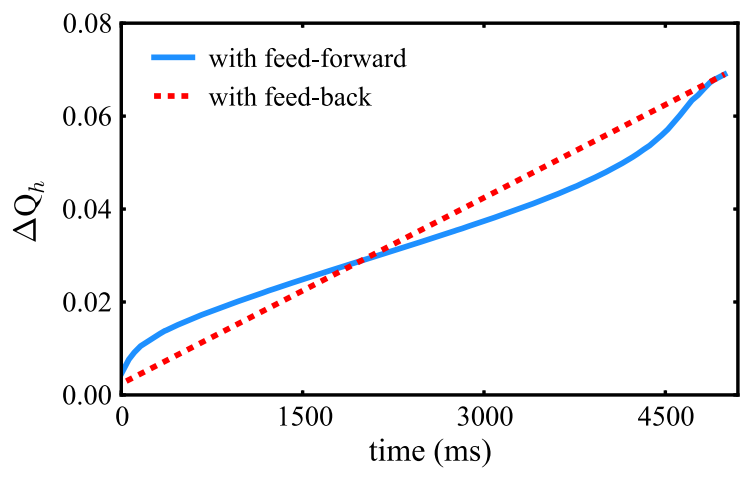

FIG. 15. Horizontal tune change along the extraction flat-top as programmed in the tune function with feed-forward (blue) and with feed-back (dashed red line) for tune sweep extraction.

due to the higher extraction momentum and hysteresis influence other cycles. Otherwise, the SPS was well reproducible. The existing infrastructure and application were reused for COSE. Instead of adjusting the $Q_{h}$ function, it is the momentum function to be trimmed for COSE. One $p$ function trim recalculates the functions of several hundred parameters, as all settings in the SPS are stored as normalized strength.

[1] M. A. Fraser et al., Slow extraction efficiency measurements at the CERN SPS, in Proc. 9th Int. Particle Accelerator Conf. (IPAC'18), Vancouver, Canada, Apr.May 2018, https://doi.org/10.18429/JACoW-IPAC2018TUPAF054.

[2] J. Beacham et al., Physics beyond colliders at CERNBeyond the standard model working group report, CERN Report No. CERN-PBC-REPORT-2018-007.

[3] F. Velotti et al., Septum shadowing by means of a bent crystal to reduce slow extraction beam loss, Phys. Rev. Accel. Beams 22, 093502 (2019).

[4] B. Goddard et al., Reduction of $400 \mathrm{GeV}$ slow extraction beam loss with a wire diffuser at the CERN SPS [Phys. Rev. Accel. Beams (to be published)].

[5] L.S. Stoel et al., Demonstration of slow extraction loss reduction with the application of octupoles at the CERN SPS, [Phys. Rev. Accel. Beams (to be published)].

[6] L. Gatignon, Physics at the SPS, Rev. Sci. Instrum. 89, 052501 (2018).

[7] W. Hardt, Ultraslow extraction out of LEAR (transverse aspects), CERN Report No. PS/DL/LEAR Note 81-6, 1981.

[8] M. Gyr, Proposal for a new servo-spill system: Power requirements for different configurations, CERN Report No. CERN-SL-95-103-BT.

[9] V. Kain, K. Cornelis, and E. Effinger, New spill control for the slow extraction in the multi-cycling SPS, CERN Report No. CERN-ACC-2016-270.

[10] M. Benedikt, Optical design of a synchrotron with optimisation of the slow extraction for hadron therapy, CERN Report No. CERN-THESIS-2000-022. 
[11] M. Tomizawa et al., Slow extraction from the J-PARC main ring using a dynamic bump, Nuclear Instrum. Methods Phys. Res., Sect. A 902, 51 (2018).

[12] L. Stoel et al., Progress toward a dynamic extraction bump for slow extraction in the CERN SPS, in Proc. 9th Int. Particle Accelerator Conf. (IPAC'18), Vancouver,
Canada, Apr.-May 2018, https://doi.org/10.18429/ JACoW-IPAC2018-TUPAF055.

[13] D. Jacquet, R. Gorbonosov, and G. Kruk, LSA-The high level application software of the LHC and its performance during the first 3 years of operation, Proc. of 14th Int. Conf. on Accelerator \& Large Experimental Physics Control Systems, San Francisco, CA, USA, 2013, pp. thppc058. 